\title{
RETRACTED ARTICLE: Predictors of institutionalization among home-dwelling older Finnish people: a 22-year follow-up study
}

\author{
Marika Salminen $^{1,2} \cdot$ Jenni Vire $^{3} \cdot$ Laura Viikari $^{3} \cdot$ Tero Vahlberg $^{4} \cdot$ \\ Hannu Isoaho $^{5} \cdot$ Aapo Lehtonen $^{3} \cdot$ Matti Viitanen $^{3} \cdot$ Seija Arve $^{6} \cdot$ Sini Eloranta ${ }^{6,7}$
}

Received: 25 September 2015/Accepted: 5 January 2016/Published online: 22 January 2016

(C) Springer International Publishing Switzerland 2016

This article has been retracted at the request of the authors and the Editors of Aging Clinical and Experimental Research. After publication of the article Salminen, M., Vire, J., Viikari, L. et al. Aging Clin Exp Res (2016). DOI:10.1007/s40520-016-0530-9, the authors realized that hazard ratios and confidence intervals in the published article are incorrect.
The online version of this article contains the full text of the retracted article as electronic supplementary material.

The authors submitted a corrected version of the manuscript (with new statistical analyses) which went through peer review process and was accepted for publication. The corrected version of this article is available at http://link. springer.com/article/10.1007/s40520-016-0722-3.
Electronic supplementary material The online version of this article (doi:10.1007/s40520-016-0530-9) contains supplementary material, which is available to authorized users.

Marika Salminen

majosa@utu.fi

1 Unit of Family Medicine, Faculty of Medicine, University of Turku, Lemminkäisenkatu 1, 20014 Turku, Finland

2 City of Turku, Welfare Division, Yliopistonkatu 30, 20101 Turku, Finland

3 Department of Geriatrics, Faculty of Medicine, University of Turku, Kunnallissairaalantie 20, 20700 Turku, Finland

4 Institute of Clinical Medicine, Biostatistics, University of Turku, Lemminkäisenkatu 1, 20014 Turku, Finland

5 Statcon Ltd., Savenvalajankatu 3 B 25, FI-20810 Turku, Finland

6 Department of Nursing Science, Faculty of Medicine, University of Turku, Lemminkäisenkatu 1, 20014 Turku, Finland

7 Hospital District of Southwest Finland, Kiinanmyllynkatu 4-8, 20521 Turku, Finland 